\title{
AusSCORE II in predicting 30-day mortality after isolated coronary artery bypass grafting in Australia and New Zealand
}

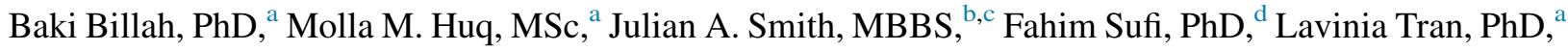 \\ Gilbert C. Shardey, MBBS, ${ }^{e}$ and Christopher M. Reid, PhD ${ }^{\mathrm{a}}$
}

\begin{abstract}
Objectives: To update the Australian System for Cardiac Operative Risk Evaluation (AusSCORE) model for operative estimation of 30-day mortality risk after isolated coronary artery bypass grafting in the Australian population.
\end{abstract}

\begin{abstract}
Methods: Data were collected by the Australian and New Zealand Society of Cardiac and Thoracic Surgeons registry from 2001 to 2011 in 25 hospitals. A total of 31,250 patients underwent isolated coronary artery bypass grafting and the outcome was 30-day mortality. A total of 2154 (6.9\%) patients had 1 or multiple missing values. Missing values were estimated assuming missing completely at random and logistic regression with a generalized estimating equation was used to address within-hospital variance. Bootstrapping methods were used to construct and validate the updated model (AusSCORE II). Also the model was validated on an out-of-creation sample of 4700 patients who underwent bypass surgery in 2012.
\end{abstract}

Results: The average age of the patients was $65.6 \pm 12.9$ years and $78.6 \%$ were male. Thirteen variables were selected in the updated model. The bootstrap discrimination and calibration of the AusSCORE II was very good (receiver operating characteristics [ROC], 82.0\%; slope calibration, 0.987). The overall observed/AusSCORE II predicted mortality was $1.63 \%$ compared with the original AusSCORE predicted mortality of $1.01 \%$. The validation of the AusSCORE II on the out-of-sample data also showed a high performance of the model (ROC, $84.5 \%$; Hosmer-Lemoshow $P$ value, .7654).

Conclusions: The AusSCORE II model provides improved prediction of 30-day mortality and successfully stratifies patient risk. The model will be useful to improve the preoperative consultation regarding risk stratification in terms of 30-day mortality. (J Thorac Cardiovasc Surg 2014;148:1850-5)

Supplemental material is available online.

The Australian System for Cardiac Operative Risk Evaluation (AusSCORE) is a 30-day mortality risk prediction model, which was developed for predicting 30-day mortality after isolated coronary artery bypass grafting $(\mathrm{CABG})$ in the Australian population. ${ }^{1}$ The AusSCORE model was published in 2009 and was developed based on 7709 patients who underwent isolated CABG from 2001 to 2007 in 6 public hospitals in the state of Victoria, Australia. However, since 2007 more hospitals

\footnotetext{
From the Departments of Epidemiology and Preventive Medicine, ${ }^{\mathrm{a}}$ and Surgery, ${ }^{\mathrm{b}}$ Monash University, Melbourne, Victoria; Department of Cardiothoracic Surgery, Monash Medical Centre, Clayton, Victoria; Department of Defense Canberra, ACT, Australia ${ }^{\mathrm{d}}$; and Cabrini Medical Centre, ${ }^{\mathrm{e}}$ Malvern, Victoria, Australia. Disclosures: Authors have nothing to disclose with regard to commercial support. Received for publication July 23, 2013; revisions received Jan 29, 2014; accepted for publication Feb 3, 2014; available ahead of print March 18, 2014.

Address for reprints: Baki Billah, $\mathrm{PhD}$, Department of Epidemiology and Preventive Medicine, Monash University, Melbourne, Victoria 3004, Australia (E-mail: baki. billah@monash.edu).

$0022-5223 / \$ 36.00$

Copyright (c) 2014 by The American Association for Thoracic Surgery

http://dx.doi.org/10.1016/j.jtcvs.2014.02.027
}

across Australia now contribute to the Australian and New Zealand Society of Cardiac and Thoracic Surgeons (ANZSCTS) registry and between 2001 and 2011 the database accumulated 53,681 cardiac surgery patients. Of these, 31,250 underwent isolated CABG. For the data from 2001 to 2011, the original AusSCORE model maintained a moderate discrimination power under the area of the receiver operating characteristics (ROC) curve but its calibration was dropped. Thus, the model failed to maintain and optimize its usefulness in contemporary cardiac surgical practice, especially in Australia and New Zealand.

In addition to reduced calibration performance of the original AusSCORE, risk model development techniques have evolved over the past few years resulting in better methods for risk prediction. Model validation improvements include bootstrapping methods that give bias-corrected accuracy indexes of the model and evaluate model specification. ${ }^{2}$ The original AusSCORE model was developed only for complete cases and the model did not address within-hospital variation.

Thus, the objectives of this study were to (1) update the original AusSCORE with a larger dataset, (2) estimate missing values, (3) address the within-hospital variation, (4) develop and validate the model using bootstrap methods 


\section{Abbreviations and Acronyms \\ AIC = Akaike Information Criteria \\ ANZSCTS $=$ Australian and New Zealand Society \\ of Cardiac and Thoracic Surgeons \\ AusSCORE $=$ Australian System for Cardiac \\ Operative Risk Evaluation

$\begin{array}{ll}\text { CABG } & =\text { coronary artery bypass grafting } \\ \text { eGFR } & =\text { glomerular filtration rate } \\ \text { EuroSCORE } & \text { European System for Cardiac } \\ & \text { Operative Risk Evaluation } \\ \text { H-L } & =\text { Hosmer-Lemoshow } \\ \text { IV } & =\text { intravenous } \\ \text { MI } & =\text { myocardial infarction } \\ \text { NYHA } & =\text { New York Heart Association } \\ \text { RAMR } & =\text { risk adjusted mortality ratio } \\ \text { ROC } & =\text { receiver operating characteristics } \\ \text { STS } & =\end{array}$

rather than split-sample methods, ${ }^{2}$ (5) evaluate model specification, and (6) develop an online calculator for 30-day mortality risk prediction.

\section{METHODS}

\section{ANZSCTS Database}

The ANZSCTS prospectively collects information on adult patients undergoing cardiac surgery in Australia. Currently, 25 hospitals across Australia and New Zealand are contributing data to the registry. Participation in the data registry is voluntary in terms of hospitals but if a hospital participates, the surgeons' participation is compulsory. The database prospectively collects 287 preoperative, intraoperative, and postoperative variables. The ANZSCTS data were collected using internationally standardized data definitions. ${ }^{1,3}$ The data collection and its audit methods have been discussed previously. ${ }^{1,4}$ The variable glomerular filtration rate had maximum missing values $(2.58 \%)$ followed by ejection fraction estimate $(2.52 \%)$, urgency of procedures $(1.11 \%)$, and 30 -day mortality $(1.01 \%)$. The remaining variables had less than $0.12 \%$ missing observations. The missing observations were estimated using multiple imputation assuming they were missing completely at random, ${ }^{12}$ The primary outcome variable for this study was 30-day mortality. The 30-day mortality status was collected by the hospital data managers by contacting patients, family members, or medical practitioners by follow-up visits or via telephone as part of clinical care.

\section{Statistical Analysis}

Stata version 11 (Stata Corporation, College Station, Tex) and R version 2.13.1 (The R Foundation for Statistical Computing) were used for data analysis. Clinical assessment along with the $\chi^{2}$ test and simple logistic regression were used to identify potential preoperative risk factors for 30 -day mortality. Statistically, a variable with $P$ value of .25 or less was considered to be a potential risk factor. A bootstrap sample of size $n$ was drawn from the original sample of the same size. The potential risk factors were entered into the multiple logistic regression model and were applied to the bootstrap samples to test the significance of the variables. A variable with a $P$ value of less than or equal to .05 was considered significant. The process was repeated 1000 times and the percentage of times that each variable appeared as significant in 1000 bootstraps was recorded.
Depending on the percentage of times the potential variables appeared as significant, 4 plausible models were developed. The final model was selected using Akaike Information Criteria (AIC), ${ }^{5}$ ROC, and Hosmer-Lemoshow (H-L) $P$ value of goodness of fit test. The first-order interaction effect and multicollinearity between clinically relevant risk factors were investigated. A generalized estimating equation was used to account for potential within-hospital variation. ${ }^{13}$ The model was validated using bootstrap methods, which provide unbiased estimates of the model's future performance (index-corrected ROC, calibration slope, and graph). ${ }^{2}$ The model was also validated for a validation sample. An online risk calculator was developed to predict a patient's preoperative risk of 30-day mortality.

\section{RESULTS \\ Patient Characteristics}

Between 2001 and 2011, the ANZSCTS database accumulated 53,681 patients who underwent cardiac surgery and, of those, $57.6 \%(\mathrm{n}=31,250)$ were isolated CABG procedures. From the list of 37 main preoperative variables, the 21 variables that were selected as potential risk factors for 30-day mortality are shown in Table 1 . The patient demographics and odds ratio for these variables along with the $P$ value and $95 \%$ confidence interval are also presented in Table 1.

Compared with the data from which the original AusSCORE was derived (in brackets) patients were similar in age $(65.6 \pm 12.9$ years $[65.7 \pm 10.1$ years] $), 78.6 \%$ were male $(76.8 \%)$, and overall 30 -day mortality was $1.63 \%$ $(1.74 \%)$. More patients were octogenarians $(7.3 \%$ [5.3\%]), on dialysis $(1.6 \%[1.3 \%])$, and had hypercholesterolemia $(81.0 \% \quad[17.9 \%])$. The definition of hypercholesterolemia has changed for the current data to include anyone being treated for hypercholesterolemia; most cardiac patients are on statins, whether or not they are actually hyperlipidemic. However, fewer patients had a severely impaired ejection fraction estimate $(4.3 \%$ [5.2\%]), New York Heart Association (NYHA) class III or IV $(20 \%$ [26.3\%]), and were classified clinically as emergency or salvage $(4.3 \%[4.6 \%])$.

\section{Risk Model}

One thousand bootstrap samples each of the size of the original sample were drawn from the original data. The 21 variables that were selected as potential predictors were entered into a multiple logistic regression model and was run on each of the bootstrap samples. The percentage of times each variable was selected as significant ( $P$ value $\leq .05)$ was recorded. The following 4 potential models were developed: model 1 , variables selected in at least $90 \%$ of bootstrap samples (age, ejection fraction estimate, previous cardiac surgery, urgency of procedures, estimated glomerular filtration rate [eGFR], NYHA class, inotrope administration, and myocardial infarction $[\mathrm{MI}])$; model 2, variables selected in at least in $80 \%$ of bootstrap samples (all variables in model 1 and 3 additional variables 
TABLE 1. Patient demographics and potential risk factors for 30-day mortality after isolated coronary artery bypass grafting

\begin{tabular}{|c|c|c|c|c|}
\hline Variable & $\begin{array}{c}\text { Prevalence \% } \\
(\mathbf{N}=\mathbf{3 1}, \mathbf{2 5 0})\end{array}$ & OR & $\begin{array}{c}P \\
\text { value }\end{array}$ & $\begin{array}{c}95 \% \\
\text { CI }\end{array}$ \\
\hline Died within $30 \mathrm{~d}(\%)$ & 1.63 & - & - & - \\
\hline Age, y mean $\pm S D$ & $65.6 \pm 12.9$ & - & - & - \\
\hline Age (ref: $<70$ y): & 61.4 & - & - & - \\
\hline $70-79$ y & 31.3 & 2.3 & $<.001$ & $1.9-2.8$ \\
\hline$\geq 80 \mathrm{y}$ & 7.3 & 4.4 & $<.001$ & $3.4-5.6$ \\
\hline Ejection fraction (ref: $>45 \%$ ): & 80.0 & - & - & - \\
\hline Moderate $(30 \%-45 \%)$ & 15.7 & 2.7 & $<.001$ & $2.2-3.4$ \\
\hline Severe $(<30 \%)$ & 4.3 & 9.1 & $<.001$ & $7.2-11.5$ \\
\hline Peripheral vascular disease & 12.2 & 2.2 & $<.001$ & $1.8-2.8$ \\
\hline Diabetic (yes) & 33.6 & 1.2 & .020 & $1.0-1.5$ \\
\hline eGFR (ref: $\geq 90 \mathrm{~mL} / \mathrm{min} / 1.73 \mathrm{~m}^{2}$ ) & 39.1 & - & - & - \\
\hline Mild $60-89 \mathrm{~mL} / \mathrm{min} / 1.73 \mathrm{~m}^{2}$ & 36.3 & 2.0 & $\begin{array}{l}<.001 \\
<.001 \\
<.001 \\
<.001\end{array}$ & $1.5-2.7$ \\
\hline Moderate $30-59 \mathrm{~mL} / \mathrm{min} / 1.73 \mathrm{~m}^{2}$ & 21.1 & 4.7 & $<.001$ & $3.6-6.1$ \\
\hline Severe $<30 \mathrm{~mL} / \mathrm{min} / 1.73 \mathrm{~m}^{2}$ & 2.1 & 9.5 & $<.001$ & $6.4-14.1$ \\
\hline Dialysis & 1.6 & 6.6 & $<.001$ & $4.0-10.8$ \\
\hline Cerebrovascular disease & 10.9 & 1.6 & $<.001$ & $1.3-2.1$ \\
\hline Respiratory disease & 12.2 & 1.5 & $<.001$ & $1.2-1.9$ \\
\hline Hypercholesterolemia & 81.0 & 0.8 & .017 & $0.6-1.0$ \\
\hline Inotrope administration & 1.6 & 14.2 & $<.001$ & $11.0-18.3$ \\
\hline NYHA class (ref: I and II) & 80.1 & - & - & - \\
\hline III & 15.3 & 2.2 & $<.001$ & $1.8-2.7$ \\
\hline IV & 6.7 & 6.9 & $<.001$ & $5.5-8.7$ \\
\hline Previous cardiac surgery & 4.5 & 2.8 & $<.001$ & $2.1-3.7$ \\
\hline Family history of CD & 42.3 & 0.7 & .003 & $0.6-0.9$ \\
\hline Cardiogenic shock & 1.7 & 15.2 & $<.001$ & $11.9-19.4$ \\
\hline Gender (female) & 21.4 & 1.9 & $<.001$ & $1.6-2.3$ \\
\hline Urgency (ref: elective) & 84.2 & - & - & - \\
\hline Urgent & 11.4 & 1.4 & .015 & 1.1-1.9 \\
\hline Emergency/salvage & 4.3 & 9.2 & $<.001$ & 7.4-11.3 \\
\hline IV nitrate administration & 7.4 & 4.9 & $<.001$ & $3.6-5.5$ \\
\hline Anticoagulant medication & 23.7 & 2.7 & $<.001$ & $2.3-3.3$ \\
\hline $\mathrm{BSA}>1.73 \mathrm{~m}^{2}$ & 82.7 & 0.5 & $<.001$ & $0.4-0.6$ \\
\hline Preoperative MI & 54.0 & 2.6 & $<.001$ & $2.1-3.2$ \\
\hline Hypertension & 78.8 & 1.4 & .006 & $1.1-1.8$ \\
\hline Preoperative resuscitation $<1 \mathrm{~h}$ & 0.9 & 12.6 & $<.001$ & 9.1-17.5 \\
\hline
\end{tabular}

$O R$, Odds ratio; $C I$, confidence interval; $e G F R$, estimated glomerular filtration rate; $N Y H A$, New York Heart Association; $C D$, cardiac disease; $B S A$, body surface area; $M I$, myocardial infarction; $I V$, intravenous; $S D$, standard deviation. See Appendix E1 for specific variable definition. See Appendix E2 for eGFR calculator.

[peripheral vascular disease, anticoagulant medication, and gender]); model 3, variables selected in at least in $60 \%$ of bootstrap samples (all variables in model 2 and 2 additional variables [cardiogenic shock and intravenous (IV) nitrate administration]); and model 4, all 21 variables in Table 1 . The ROC, H-L $P$ value, and AIC for these models are respectively as follows: model $1,81.8 \%, 32.7 \%$, and 3965 ; model $2,82.2 \%, 32.4 \%$, and 3943 ; model 3 , $82.4 \%, 60.1 \%$, and 3936 ; model $4,82.3 \%, 45.0 \%$, and 3588. The H-L $P$ value does not necessarily increase with increasing number of variables in the model. Model 3
TABLE 2. 30-day mortality risk prediction model: AusSCORE II

\begin{tabular}{|c|c|c|c|}
\hline Variable & OR & $P$ value & $95 \% \mathrm{Cl}$ \\
\hline Age (ref: $<70$ y) & - & - & - \\
\hline $70-79 y$ & 1.6 & $<.001$ & $1.5-1.8$ \\
\hline$\geq 80 \mathrm{y}$ & 2.6 & $<.001$ & $2.4-2.9$ \\
\hline Ejection fraction (ref: $>45 \%$ ) & - & - & - \\
\hline Moderate $(30 \%-45 \%)$ & 1.7 & $<.001$ & $1.5-1.9$ \\
\hline Severe $(<30 \%)$ & 3.6 & $<.001$ & $3.1-4.2$ \\
\hline Previous cardiac surgery & 2.3 & $<.001$ & $1.9-2.8$ \\
\hline eGFR (ref: $\geq 90 \mathrm{~mL} / \mathrm{min} / 1.73 \mathrm{~m}^{2}$ ) & - & - & - \\
\hline $60-89 \mathrm{~mL} / \mathrm{min} / 1.73 \mathrm{~m}^{2}$ & 1.5 & .003 & $1.2-2.0$ \\
\hline $30-59 \mathrm{~mL} / \mathrm{min} / 1.73 \mathrm{~m}^{2}$ & 2.0 & $<.001$ & $1.7-2.4$ \\
\hline$<30 \mathrm{~mL} / \mathrm{min} / 1.73 \mathrm{~m}^{2}$ & 3.8 & $<.001$ & $2.8-5.1$ \\
\hline Dialysis & 3.9 & $<.001$ & $2.4-6.3$ \\
\hline Urgency (Ref: elective) & - & - & - \\
\hline Urgent & 1.1 & .190 & $0.9-1.4$ \\
\hline Emergency/salvage & 2.6 & $<.001$ & $2.1-3.1$ \\
\hline NYHA class (ref: I and II) & - & - & - \\
\hline III & 1.4 & $<.001$ & $1.2-1.6$ \\
\hline IV & 1.8 & $<.001$ & $1.5-2.1$ \\
\hline Inotrope administration & 2.1 & $<.001$ & $1.8-2.6$ \\
\hline Peripheral vascular disease & 1.5 & $<.001$ & $1.3-1.7$ \\
\hline Previous MI & 1.5 & $<.001$ & $1.4-1.6$ \\
\hline Anticoagulant medication & 1.3 & .019 & $1.0-1.6$ \\
\hline Gender (female) & 1.5 & $<.001$ & $1.3-1.6$ \\
\hline Cardiogenic shock & 1.8 & $<.001$ & $1.4-2.3$ \\
\hline IV nitrates administration & 1.5 & $<.001$ & $1.2-1.8$ \\
\hline
\end{tabular}

$O R$, Odds ratio; $C I$, confidence interval; $e G F R$, estimated glomerular filtration rate; NYHA, New York Heart Association; $M I$, myocardial infarction; $I V$, intravenous.

with 13 variables was selected as the final model (AusSCORE II) by comparing ROC, H-L $P$ value, and AIC between the 4 potential models. The variables in the final model are age, gender, ejection fraction estimate, previous cardiac surgery, urgency of procedures, eGFR, NYHA class, inotrope administration, MI, peripheral vascular disease, anticoagulant medication, cardiogenic shock, and IV nitrate administration. There is no multicollinearity or first-order interaction effect between the risk factors in the model. Among the variables in the final risk model, IV nitrate administration was selected in the least number $(66.5 \%)$ of bootstrap samples. No variables were selected in between $30 \%$ and $65 \%$ of the samples. Hence, variables that were significant in less than $30 \%$ of the samples were excluded from the final model despite some of them occurring often in patients undergoing $\mathrm{CABG}$.

The risk factors in the AusSCORE II model and their corresponding odds ratio, $95 \%$ confidence interval, and $P$ value are presented in Table 2. The key drivers for 30-day mortality after CABG were age 80 years or more, ejection fraction less than $30 \%$, previous cardiac surgery, eGFR less than $30 \mathrm{~mL} / \mathrm{min} / 1.73 \mathrm{msq}$ (excludes dialysis), on dialysis, having an emergency or salvage procedure, NYHA class IV, and inotrope administration. Women had a higher risk than men. 

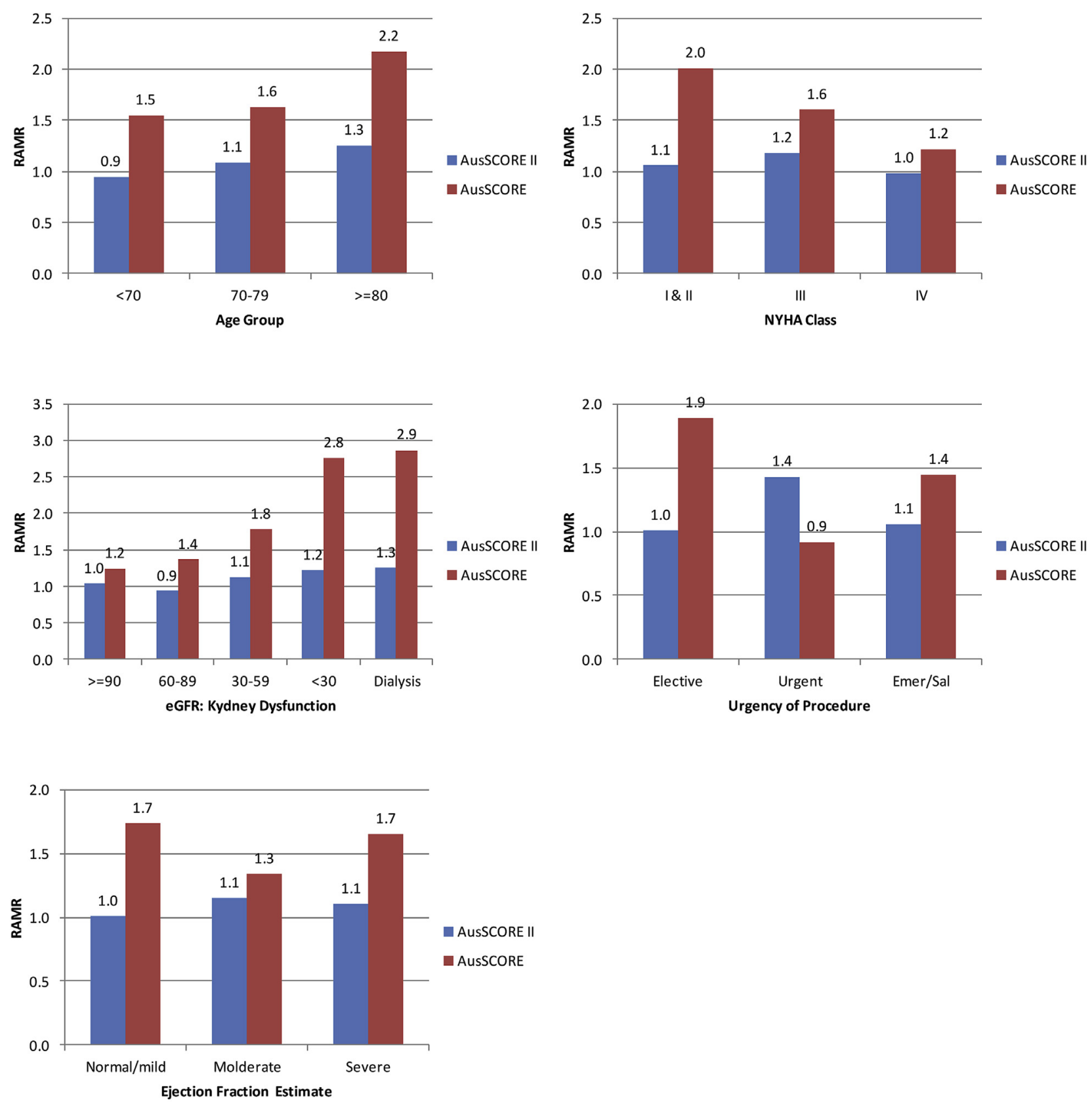

NYHA: New York Heart Association, eGFR: Estimated glomerular filtration rate.

FIGURE 1. Comparison of risk adjusted mortality ratio (RAMR) between AusSCORE II and the original AusSCORE. NYHA, New York Heart Association; $e G F R$, estimated glomerular filtration rate; AusSCORE, Australian System for Cardiac Operative Risk Evaluation.

\section{Prediction Performance}

The overall prediction of 30-day mortality for the AusSCORE II model compared with the original AusSCORE model was as follows: AusSCORE II/observed, $1.63 \%$; original AusSCORE, $1.01 \%$. The overall risk adjusted mortality ratio (RAMR, observed/ expected) was 1.61 for the original AusSCORE. Thus, the original AusSCORE model underestimated the mortality risk and hence had reduced calibration for the current data.
The optimally corrected ROC $(82.0 \%)$ and slope calibration (0.987) for AusSCORE II were very high, demonstrating a successful logistic model. The bootstrap calibration curve presented in Figure E1 also shows an excellent calibration especially for low to high-risk patients. When validated on the original data, the discrimination of AusSCORE II was better than the original AusSCORE (within brackets): ROC, 81.9\% (78.28\%). AusSCORE II calibrated well (H-L $P$ value .4232) but the 
original AusSCORE (H-L $P$ value $<.001$ ) had poor calibration. The RAMR for some specific risk factors is presented in Figure 1, again demonstrating good calibration for the AusSCORE II model. The original AusSCORE model, however, had a poorer calibration and strong tendency to underestimate 30-day mortality.

When validated on the 2012 data, both models showed a very good discrimination power, however the original AusSCORE model had a poor calibration (AusSCORE II, ROC $84.5 \%$, H-L $P$ value .7654; AusSCORE, ROC $84.3 \%$, H-L $P$ value .0019). Furthermore, the calibration of the AusSCORE II model, when examined by comparing mean observed and predicted mortalities for each decile of the predicted values (Figure E2), demonstrates that in general the model was well calibrated.

The discrimination power of the AusSCORE II was also compared with that of the EuroSCORE II and the Society of Thoracic Surgeons (STS) model and it was found that the ROC of AusSCORE II $(82 \%)$ was competitive with that of the EuroSCORE II $(80.95 \%)$ and STS $(81.2 \%)$ models when they were validated on their own data.

\section{Model Specification}

The 1000 bootstraps for variable selection for AusSCORE II was repeated 10 times to evaluate the model specification (or variable uncertainty). The variation in the percentage of times that each of the 21 potential variables was selected in each of the 10 sets of 1000 bootstrapping was insignificant and, hence, in each repeat the same set of variables was selected in the AusSCORE II model suggesting a stable model.

\section{Online Calculator and Case Study}

An online calculator was developed to predict patient's 30-day mortality and risk group. Consider a patient who was female, 76 year old, classified as NYHA class III, had eGFR $80 \mathrm{~mL} / \mathrm{min}$, and ejection fraction $40 \%$. The patient underwent isolated CABG on an elective basis. Using the calculator, the patient's 30-day mortality risk was $1.53 \%$ (Figure E3).

\section{DISCUSSION}

The original AusSCORE model was published in 2009 and was developed based on the Australian Society of Cardiac and Thoracic Surgeons data collected from 6 public hospitals between 2001 and 2007 in the state of Victoria, Australia. Since the development of the original AusSCORE model, the plan was to update the model after the completion of 20,000 isolated CABGs. The database currently includes data from 25 private and public hospitals across Australia and now includes 53,681 patients, of whom 31,250 underwent isolated CABG. Thus, these data are now more representative of the Australian population compared with the dataset used to derive the original AusSCORE model.

Model specification, which greatly depends on the uncertainty in the variables in the model, should be a concern in any risk modeling. ${ }^{2}$ However, this has not been addressed for risk modeling in the literature. The model specification for AusSCORE II was evaluated using multiple sets of bootstrapping. The results showed that the uncertainty in the variable selection in the AusSCORE II model was minimal, suggesting the model was specified adequately.

The original AusSCORE and other risk models in the literature, including EuroSCORE II and the STS model, were developed based on the split-sample method. The split-sample method affects a model's predictive accuracy and precision of the estimates of the accuracy. AusSCORE II, however, was developed on an entire dataset and was validated using the bootstrap method. The bootstrap method uses the full dataset for model development and model validation. This method provides the bias-corrected discriminatory and calibration power of the model. The bootstrap method gives slope calibration as opposed to the H-L $P$ value; the latter method has some inherent limitations. ${ }^{6-8}$ Addressing within-hospital variation and missing value estimation using multiple imputation was another key addition to the AusSCORE II model.

In the original AusSCORE model, serum creatinine $(\mathrm{mg} / \mathrm{dL})$ level was used as a measure of kidney dysfunction, however eGFR was used in the AusSCORE II model. The eGFR depends on the patient's age, gender, weight, and serum creatinine level $(\mathrm{mg} / \mathrm{dL})$ and is a better indicator of kidney dysfunction. " Also the category "urgent" for "urgency of procedures" was tightened whereby a patient was classified as "urgent" if the surgery took place within 72 hours of catheterization.

In AusSCORE II, 13 variables were identified as the key risk factors compared with 8 variables in the original AusSCORE model. ${ }^{1}$ The new variables in the AusSCORE II model were gender, previous MI, inotrope administration, IV nitrate administration, anticoagulant medication, and cardiogenic shock. This showed that the patient's preoperative risk has increased since the development of the original AusSCORE model. However, the overall 30-day mortality has dropped perhaps because of improvement in cardiac surgery techniques, early intervention, and increased postoperative care. The variable hypercholesterolemia was significant in the original AusSCORE model but was not significant in the AusSCORE II. This may be a reflection of the increased use of statin medication across the population in the current database. The diabetic status of a patient was significant in the simple logistic regression, however was insignificant when adjusted for other potential risk factors. This may be because diabetic status is associated with peripheral 
vascular disease, cerebrovascular disease, and renal dysfunction. Being female was a risk factor in the AusSCORE II model, which is supported by the EuroSCORE II and STS models. ${ }^{6,7,10}$

Compared with the original AusSCORE, AusSCORE II was a better predictor of 30-day mortality. The original AusSCORE model maintained good discrimination but its calibration was poorer for the current data. The original AusSCORE model underpredicts mortality for this population. This may be because the AusSCORE II model is more representative of the population or because of improvements in model development and validation methods. ${ }^{2}$

The original EuroSCORE model did not accurately predict outcomes in the Australian population. ${ }^{11}$ The EuroSCORE II and STS models could not be validated for the Australian cohort because of differences in data definition between these registries. For example, the variables extracardiac arteriopathy, chronic pulmonary dysfunction, neurologic or musculoskeletal dysfunction, critical, pulmonary artery systolic pressure, and thoracic aorta in the EuroSCORE II model are not available in the ANZSCTS registry. It would be ideal if consensus could be reached regarding unique data definitions across populations that would assist in model standardization and benchmarking. A study is inevitable to address all data definition differences between the major international data registries. The discriminatory power of the AusSCORE II model was found to be as good as or better than most of the risk models in the literature, including the original AusSCORE, EuroSCORE II, and STS models, when they were applied to their own data. ${ }^{6,7,10}$

The online calculator was an important addition to the Australian 30-day mortality risk prediction after isolated CABG. This is the first attempt to evaluate a patient's preoperative risk of mortality online; which can be used to calculate logistic (absolute) risk of 30-day mortality.

\section{Limitations}

The conversion of continuous age to categorical is a limitation of this study. However, the interpretation of risk associated with categorical variables is easy to follow by cardiologists, cardiac surgeons, and other medical professionals in preference to continuous variables. Furthermore, this type of model can easily be validated on any external data, which makes the model user friendly.

\section{CONCLUSIONS}

AusSCORE II is the updated version of the original AusSCORE model, which will help to improve preoperative decision making, as well as risk stratification and patient counseling.

\section{References}

1. Reid C, Billah B, Dinh D, Smith J, Skillington P, Yii M, et al. An Australian risk prediction model for 30-day mortality after isolated coronary artery bypass: the AusSCORE. J Thorac Cardiovasc Surg. 2009;138:904-10.

2. Harrell FE. Regression modeling strategies: with applications to linear models, logistic regression, and survival analysis. New York, London: Springer; 2001.

3. Reid CM, Rockell M, Skillington PD, Shardey GC, Smith JA, Yii M, et al. Initial twelve months experience and analysis for 2001-2002 from the Australasian Society of Cardiac and Thoracic Surgeons, Victorian Database Project. Heart Lung Circ. 2004;13:291-7.

4. Billah B, Reid CM, Shardey GC, Smith JA. A preoperative risk prediction model for 30-day mortality following cardiac surgery in an Australian cohort Eur J Cardiothorac Surg. 2010;37:86-92.

5. Akaike H. Statistical predictor identification. Ann Inst Math Stat. 1970;22 203-17.

6. Shahian DM, O'Brien SM, Filardo G, Ferraris VA, Haan CK, Rich JB, et al The Society of Thoracic Surgeons 2008 cardiac surgery risk models: part 3 valve plus coronary artery bypass grafting surgery. Ann Thorac Surg. 2009;88(Suppl): S43-62.

7. Shahian DM, O'Brien SM, Filardo G, Ferraris VA, Haan CK, Rich JB, et al. The Society of Thoracic Surgeons 2008 cardiac surgery risk models: part 1 coronary artery bypassgrafting surgery. Ann Thorac Surg. 2009;88(Suppl): S2-22.

8. Marcin JP, Romano PS. Size matters to a model's fit. Crit Care Med. 2007;35: 2212-3.

9. Cockcroft DW, Gault MH. Prediction of creatinine clearance from serum creatinine. Nephron. 1976;16:31-41

10. Nashef SA, Roques F, Sharples LD, Nilsson J, Smith C, Goldstone AR, et al. EuroSCORE II. Eur J Cardiothorac Surg. 2012;41:734-44; discussion 744-5.

11. Yap C-H, Reid C, Yii M, Rowland MA, Mohajeri M, Skillington PD, et al Validation of the EuroSCORE model in Australia. Eur J Cardiothorac Surg. 2006;29:441-6.

12. Royston P, White IR. Multiple imputation by chained equations (MICE) Implementation in Stata. J Stat Softw 2011;45(4).

13. Hartley JA, Negassa A, Edwardes MD, Forrester JE. Statistical analysis of correlated data using generalized estimating equations: an orientation. Am J Epidemiol. 2003;157:364-75. 


\section{APPENDIX E1. DESCRIPTION OF KEY VARIABLES}

Estimated glomerular filtration rate (eGFR): estimates how much blood passes through the tiny filters in the kidneys, called glomeruli, each minute. Kidney function is classified as normal (eGFR $\geq 90 \mathrm{~mL} / \mathrm{min} /$ $1.73 \mathrm{msq}$ ), mild (eGFR $60-89 \mathrm{~mL} / \mathrm{min} / 1.73 \mathrm{msq}$ ), moderate (eGFR $30-59 \mathrm{~mL} / \mathrm{min} / 1.73 \mathrm{msq}$ ), severe (eGFR $\leq 29 \mathrm{~mL} / \mathrm{min} / 1.73 \mathrm{msq}$ ), and dialysis. The stages normal to severe exclude dialysis.

Urgency of procedures: elective, the procedure could be deferred without increased risk of compromised cardiac outcome; urgent, the patient had the surgery within 72 hours of catheterization; emergency, unscheduled surgery required in next available theatre on same day because of refractory angina or cardiac compromise; salvage, the patient is undergoing cardiopulmonary resuscitation en route to the operating room, that is, before surgical incision.

Cardiogenic shock: is the patient, at the time of procedure, in a clinical state of hypoperfusion according to either of the following criteria: (1) systolic blood pressure $80 \mathrm{~mm} \mathrm{Hg}$ and/or cardiac index 1.8 despite maximal treatment; (2) IV inotropes and/or intra-aortic balloon pump necessary to maintain systolic blood pressure $>80 \mathrm{~mm} \mathrm{Hg}$ and/or cardiac index $>1.8$.

Nitrate administration: patient on IV nitrates on day of surgery (on drug when entered operation).

Inotrope administration: any inotrope use to maintain cardiac output for longer than 4 hours postoperatively (includes dopamine at $>300 \mu \mathrm{g} / \mathrm{min}$ ). Note: routinely administered milrinone does not count.

Anticoagulant medication: patient given warfarin/ heparin/low molecular weight heparinoid $\leq 24$ hours before surgery.

Peripheral vascular disease: whether the patient has peripheral vascular disease, as indicated by claudication either with exertion or at rest; amputation for arterial insufficiency; aorto-iliac occlusive disease reconstruction; peripheral vascular bypass surgery, angioplasty, or stent; documented abdominal aortic aneurysm, abdominal aortic aneurysm repair, or stent; positive noninvasive testing documented.

\section{APPENDIX E2. CALCULATION OF EGFR}

eGFR was calculated using the Cockroft-Gault formulae ${ }^{9}$ where the creatinine level was adjusted for the patient's age, weight, and gender:

$$
\begin{aligned}
\text { eGFR }= & (\text { weight }[\mathrm{kg}] \times(140-\text { age }[\text { years }])) \\
& \times \text { gender } /(72 \times \text { serum creatinine }[\mathrm{mg} / \mathrm{dL}])
\end{aligned}
$$

where gender $=1$ for male and 0.85 for female.

\section{Prediction Model}

The following multivariable logistic regression model was used for 30-day mortality risk prediction:

$$
\text { Mortality risk }=\frac{\exp \left(\mathrm{b}_{0}+\sum \mathrm{bX}+\sum \mathrm{b}^{*} \mathrm{X}^{*}\right)}{1+\exp \left(\mathrm{b}_{0}+\sum \mathrm{bX}+\sum \mathrm{b}^{*} \mathrm{X}^{*}\right)}
$$

where $b_{0}$ is the baseline effect and $\sum b X$ is the sum of $\beta$ coefficients for all risk factors (categorical) present in the patient $(X=1$ if the risk factor is present and $X=0$ if absent). For each continuous covariate, the $\beta$ coefficient is multiplied by the value of the covariate and then added up for all covariates. This add up value is denoted by $\sum b^{*} X^{*}$ in this model. In the AusSCORE II model, there is no continuous covariate, hence the term $\sum b^{*} X^{*}$ was dropped from the model.

\section{Risk Prediction}

The AusSCORE II model can be used to calculate the logistic risk for 30-day mortality for each patient undergoing isolated $\mathrm{CABG}$. This risk should be adjusted and then reported to the patient. ${ }^{10}$ The calculated risk for a patient can be adjusted using the following formula:

$$
\begin{aligned}
\text { Adjusted risk }= & \frac{\text { Surgeon's or unit's observed mortality }}{\text { Surgeon's or unit's predicted mortality }} \\
& \times \text { Patient's predicted logistic risk }
\end{aligned}
$$

This formula for individual patient's risk prediction is appropriate if the unit (hospital) or the respective surgeon has performed a large number of procedures. ${ }^{10}$ However, for a unit with a smaller number of patients, the calculated logistic risk can be adjusted by the overall mortality ratio. 


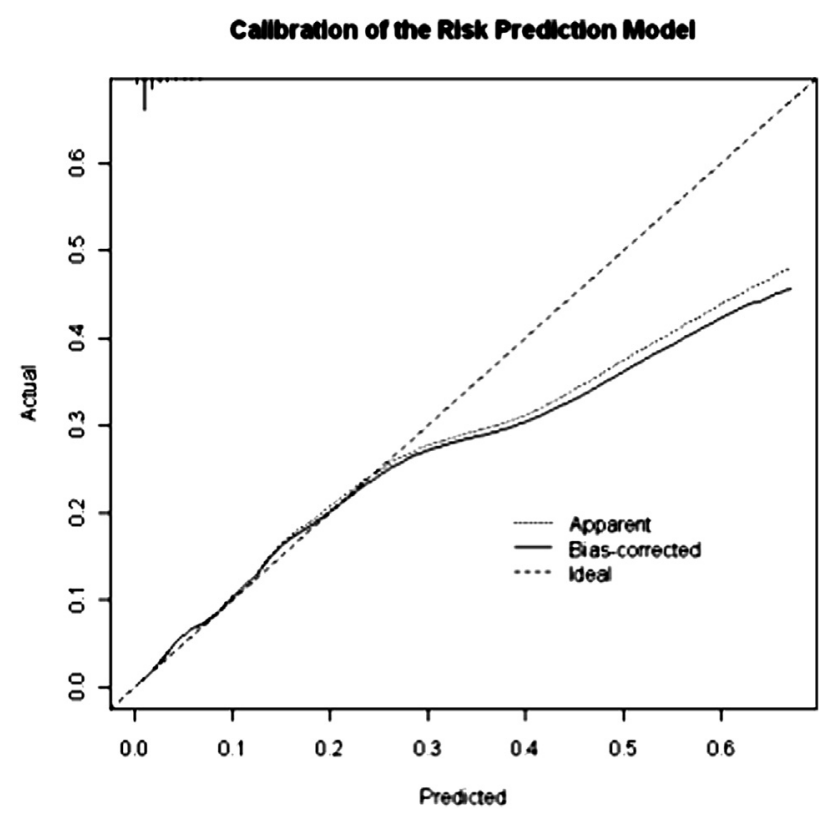

FIGURE E1. Bootstrap calibration of the AusSCORE II model.
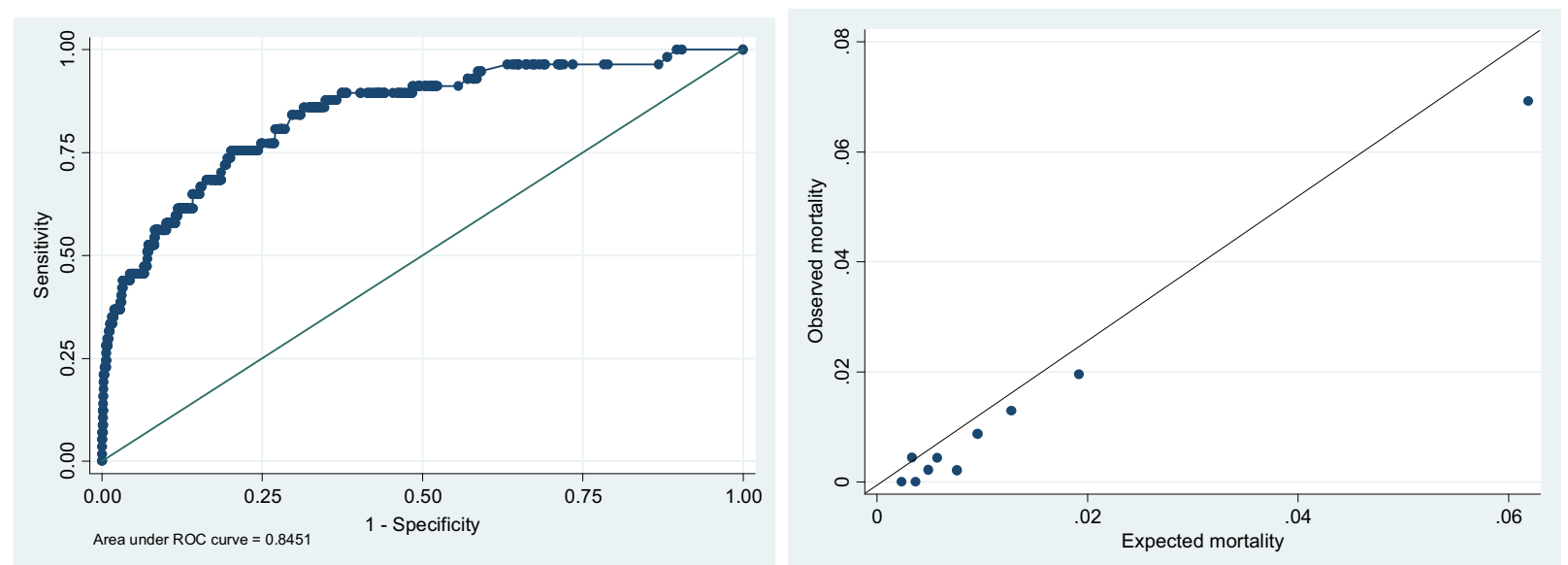

FIGURE E2. Validation of AusSCORE II for the 2012 data. ROC, Receiver operating characteristics.

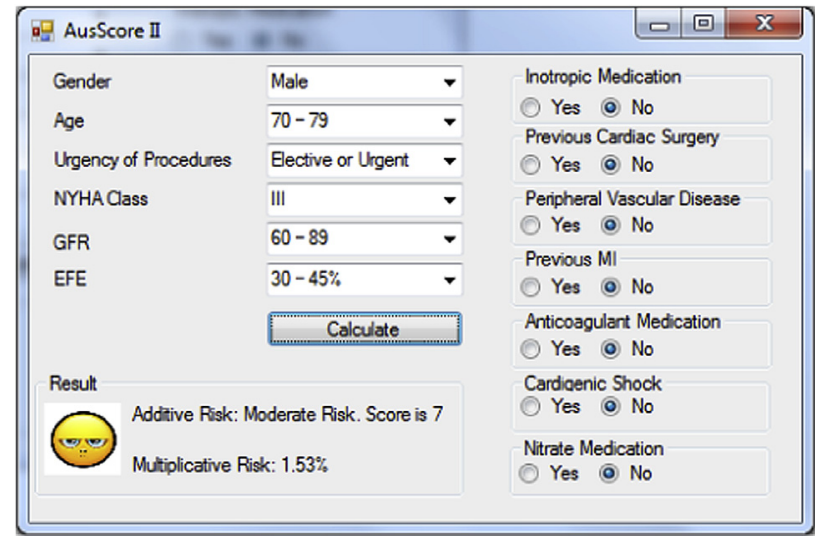

FIGURE E3. Online calculator for preoperative risk of 30-day mortality after coronary artery bypass grafting. NYHA, New York Heart Association; $G F R$, glomerular filtration rate; $E F E$, ejection fraction estimated. 